\title{
Beating of monopole modes in nuclear dynamics.
}

\author{
D. Lacroix and Ph. Chomaz \\ G.A.N.I.L, B.P. 5027, F-14076 Caen Cedex 5, France.
}

March 22, 2018

\begin{abstract}
Time-dependent Hartree-Fock simulations of the evolution of excited gold fragments have been performed. The observed dynamics appears more complex than the collective expansion picture. The minimum density is often not reached during the first density oscillation because of the beating of several collective compression modes.
\end{abstract}

\section{Introduction}

Studying the dynamics of a highly excited nucleus, such as those created in Heavy-ion collisions, is a priori a hard task since we are dealing with a many-body quantal problem involving a large number of degrees of freedom. A widely used approximation is to reduce the problem to the dynamics of few collective variables, the remaining degrees of freedom being treated in a statistical manner. In particular, excited nuclear systems are often assumed to simply be hot nuclei expanding or contracting isentropically in a unique collective compression mode, the so-called breathing mode ( see for example the expanding fireball scenario or the fragmentation models [1], 2, 3]).

This picture has been recently applied to explain the GSI data concerning the correlation between the apparent temperature and the excitation energy of fragmentation events known as the Aladin "caloric-curve" (see ref. [目] and refs. therein). In the approach of ref. [⿴囗⿴囗十) the fragmentation of various nuclear systems formed by the projectile remains after a collision, is assumed to occur 
at the respective turning points of the induced giant breathing mode. The location of these turning points in temperature and density is proposed to be the explanation of the observed "caloric-curve" [5]. More generally, most of the multifragmentation data are understood assuming that the system expands to reach a low density value either in statistical models [6] or in dynamical simulations [7] using stochastic approaches [8, 9]. The general belief is that the system breaks during the first expansion associated with the giant breathing mode of the hot nucleus. More generally, this assumption is often made in simple semi-classical simulations or in multifragmentation models [1, 2, 3.

However, recent results about photon production during Heavy-Ion collisions seems not to be compatible with this simple scenario of a fast multifragmentation during the first expansion of the system and the data have been understood assuming at least one recompression before break-up [10, 11].

On the other hand, from the study of monopole vibrations, it is wellknown that nuclei can exhibit a large variety of compression modes 12, 13. Moreover, microscopic description (using TDHF or is linear version) of monopole vibrations predicts that these modes cannot be simply described by the breathing mode picture. Since these giant resonances are the small amplitude limit of the collective expansion assumed to occur in heavy ion collisions, one may worry if the picture of a unique large amplitude collective breathing mode leading the hot nuclei to low density regions is not too simple. Therefore, in this article, we propose to study the large amplitude motion of an excited nucleus within the framework of the Time-Dependent Hartree-Fock (TDHF) theory in order to critically discuss the validity of the single breathing mode picture. It should be noticed that, heavy ion collisions are rather well described by simulations using the semi-classical version of TDHF: the Vlasov equation, possibly extended to take into account the damping coming from nucleon-nucleon collisions (i.e. the so-called BUU approach 14 ). In these simulations the expansion phase appears to be dominated by the meanfield dynamics confirming that TDHF can be a good tool to investigate this large amplitude motion. Furthermore, as far as we know, TDHF is the only well-tested quantum model tractable for Heavy-Ion collisions. Of course, a quantum model that include collision effects (extended TDHF) would be preferable. However, we believe that the introduction of this effect will not change considerably the discussion since, during the first expansion, the gain and lost term of two-body collisions are almost equal and so cancel.

In this article, we will analyzed the TDHF dynamics and we will point 
out that some aspects, important for the dynamics, cannot be accounted for in the simple breathing mode picture. This may change our understanding of the expansion dynamics of nuclei created during heavy ion collisions. In particular, we observe that, in general, the minimum central density is not reached during the first density oscillation. This may allow the system to undergo several monopolar oscillations before breaking in pieces as the photon data are suggesting.

\section{Mean-Field dynamics}

In mean-field evolution all the information is contained in the single-particle density operator $\hat{\rho}$. Let us introduce the single-particle basis which diagonalizes this operator: $\hat{\rho}=\sum\left|\Phi_{\beta}>n_{\beta}<\Phi_{\beta}\right|$, where $\beta$ contains all quantum numbers. Following the work of ref. [15], we consider the mean-field evolution of a spherical symmetric , spin-isospin saturated nucleus. The mean-field is parameterized as

$$
U(r)=\frac{3}{4} t_{0} \rho(r)+\frac{(\sigma+2)}{16} \rho(r)^{\sigma+1}+V_{0} \int \frac{\exp \frac{\left|r-r^{\prime}\right|}{a}}{\frac{\left|r-r^{\prime}\right|}{a}} \rho\left(r^{\prime}\right) d r^{\prime}+V_{C}
$$

The two first terms are the same as in ref.15 and a general discussion could be found in [16]. The Yukawa potential is introduced in order to mimic the gradient part of the potential as in ref. 17, 18. Finally, $V_{C}$ is the direct Coulomb potential which is, in our calculation, approximately treated by given a charge $\frac{Z}{A}$ to each nucleon. Parameters are chosen in order to have the same property as the $S k M^{*}[19$ force for the nuclear matter. We have taken $t_{0}=-2191.73 \mathrm{MeV} \mathrm{fm}^{3}, t_{3}=18818.8 \mathrm{MeV} \mathrm{fm}^{7 / 2}, \sigma=\frac{1}{6}, a=0.45979 \mathrm{fm}$, $V_{0}=-461.07 \mathrm{MeV} \mathrm{fm}^{3}$. For the nuclear matter, this leads to $E / A=-15.8$ $\mathrm{MeV}$ at a saturation density $\rho_{\infty}=0.16 \mathrm{fm}^{-3}$ and to an incompressibility modulus of $K_{\infty}=200 \mathrm{MeV}$ which corresponds to a soft Equation Of State. It should be noticed that the presented results do not qualitatively depend upon the compressibility of the nuclear force.

\footnotetext{
${ }^{1}$ Either experimental evidence, for example in central events selected with INDRA, or theoretical simulation of the BUU type confirms the fact that a spherical equilibrated source can be a reasonable ansatz.
} 
Since in TDHF, the initial symmetry of the system is conserved, the dynamical evolution reduces to a set of equations for the radial part $\varphi_{n l}(r, t)$ of the single-particle wave functions $\Phi_{\beta}(\vec{r}, t)$

$$
i \hbar \frac{\partial}{\partial t} \varphi_{n l}(r)=\left\{\frac{-\hbar^{2}}{2 m} \frac{\partial}{\partial r^{2}}+\frac{\hbar^{2} l(l+1)}{2 m r^{2}}+U(\rho(r, t))\right\} \varphi_{n l}(r)
$$

where $n$ and $l$ are the principal and orbital numbers and the density takes the particular form

$$
\rho(r, t)=4 \sum_{n, l}(2 l+1) n_{n l} \frac{\left|\varphi_{n l}(r, t)\right|^{2}}{4 \pi r^{2}},
$$

Numerically, we consider 40 orbitals, $\varphi_{n l}$. The Schrödinger equation is solved in coordinate representation. The lattice of size $300 \mathrm{fm}$ is discretized in steps of size $\Delta r=0.2 \mathrm{fm}$. An imaginary time method is used to generate the ground state of the static problem [15, 16] ( a small external field $\lambda r^{2}$ with $\lambda=0.25 \mathrm{MeV} \mathrm{fm}^{-2}$ is added during this initialization in order to define the particle states). Occupation numbers are calculated afterwards according to the Fermi-Dirac distribution at the considered temperature. We perform the TDHF evolution up to $1500 \mathrm{fm} / \mathrm{c}$ with a time step of $0.75 \mathrm{fm} / \mathrm{c}$.

In order to study nuclei comparable to those expected to be produced in reactions, we take the same initial conditions as in ref. [4] for the fragments of a Au-projectile. Let us first focus on one typical initial condition : an excited fragment of mass $A=191$. The excitation energy is taken from the abrasionablation model[20]. Here we consider that the excitation energy is related to the number $(\Delta A)$ of particles lost during the abrasion-ablation stage of the collision by the relation $E^{*}=13.3 \Delta A \mathrm{MeV}$. Furthermore, following ref. [4] we assume that the projectile fragments are initially slightly dilated. We implement this dilution by rescaling the wave-function according to $r \rightarrow$ $r 0.8^{-1 / 3}$. In this condition the temperature is adjusted in order to obtain the correct excitation energy. For the considered fragment which is closed from the projectile, the excitation energy is small and partly stored in the initial dilution so that the temperature is only $T=0.55 \mathrm{MeV}$. As we will discuss in the following, the conclusion we will draw are rather general and the complex dynamics is observed for a wide range of initial value of the mass, the density and the temperature. 


\section{Results}

\subsection{Density profiles}

In fig. 1] we display the density (solid line) at various times as predicted by the mean-field approximation?. We observe that the density profile display a lot of distortions. To emphasize this point we have fitted the density profile at each time by a Fermi shape

$$
\rho_{F}(r, t)=\frac{\rho(t)}{1+\exp \left(\frac{r-R(t)}{a(t)}\right)}
$$

Typical results of the fit are displayed in fig.1(dashed curves). At initial time, the density profile is well fitted by a Fermi shape. In a scaling picture of the evolution, one expects, at all time, that this fit should be also good. The strong difference between the fitted Fermi shape and the real density profile at time $t=108 \mathrm{fm} / \mathrm{c}$ demonstrates how far we are from a simple scaling picture of the expansion.

From the three parameters $\rho(t), R(t)$ and the diffuseness coefficient $a(t)$, we can define three scaling parameters as

$$
\alpha_{R}=\frac{R(t)}{R\left(t_{0}\right)} \quad \alpha_{a}=\frac{a(t)}{a\left(t_{0}\right)} \quad \alpha_{\rho}=\left(\frac{\rho\left(t_{0}\right)}{\rho(t)}\right)^{\frac{1}{3}}
$$

where $t_{0}$ is the initial time. If the collective dynamics is dominated by a unique breathing mode (a global scaling of the density profile) the three $\alpha$ should be equal and should oscillate with a unique frequency close to the one of the monopolar vibration of the considered nucleus. ${ }^{3}$

In fig.2 (Top), we show the evolution of the $\alpha$ 's as a function of time. From this figure, it is clear that only $\alpha_{R}$ and $\alpha_{\rho}$ display regular oscillation with a given frequency. Performing a Fourier transform of this quantities, we found a marked peak at $\hbar \omega=12.5 \mathrm{MeV}$ (which is around the expected breathing mode frequency of the considered nucleus at zero temperature).

\footnotetext{
${ }^{2}$ It should be noticed that we have checked that putting the ingredients considered in reference [13] we recover their results. The difference is that we are making a more detailed analysis for a heavier nucleus.

${ }^{3} \mathrm{~A}$ possible shift in frequency can be due to the anharmonicity of the large amplitude motion and to the effect of the temperature.
} 
On contrary, $\alpha_{a}$ contains clearly a complex superposition of many modes and is never comparable to the two other scaling factors. The Fourier transform of $\alpha_{a}$ presents not only the frequency $\hbar \omega=12.5 \mathrm{MeV}$ but also two other broad peaks around $17 \mathrm{MeV}$ and $26 \mathrm{MeV}$, and a small peak around $40 \mathrm{MeV}$. The Fermi shape fit being unrealistic for the density profile at time $t=108 \mathrm{fm} / \mathrm{c}$, it is difficult to really conclude on the link between the presence of different collective modes and the appearance of hollow shapes. In the following, we will characterize more precisely this link.

From fig. 1 it is clear that not only the surface of the nucleus is following a complex dynamics but also the interior which exhibits a tendency to produce hollow structures. To quantify this feature, we have computed two different averaged densities as a function of time: the solid line in figure 2 (bottom) represents the averaged density in a sphere of $2 \mathrm{fm}$ radius $\left(\rho_{2 f m}\right)$ whereas the dashed line stands for the density averaged in a sphere of $5 \mathrm{fm}\left(\rho_{5 f m}\right)$. The sphere of radius $5 \mathrm{fm}$ is big enough to contain most of the volume of the nucleus and small enough (see figure 1) to not be polluted by the surface shape. As expected, $\rho_{5 f m}$ presents a regular oscillation at the breathing mode frequency. On contrary, it is clear that $\rho_{2 f m}$ contains more than the breathing mode, in particular, this quantity is more sensitive to the presence of hole at the center of the nucleus as seen in fig.1. Therefore, it appears that in addition to the expected large amplitude volume mode other modes affecting both the interior and the surface of the nucleus are present in a complete TDHF simulation.

\subsection{Collective vibrations}

In order to quantitatively analyze the observed complex oscillation pattern of the density, we have performed the Fourier transform of $\rho(r, t)$ leading to the spectral density $\tilde{\rho}(r, \omega)$. The Fourier transform is performed up to $1500 \mathrm{fm} / \mathrm{c}$ which is sufficient to have a resolution of few hundred keV. A plot of $|\tilde{\rho}(r, \omega)|$ for $\rho / \rho_{0}=0.8$ is displayed in fig. 3(Top). We see in particular that non-local waves develop during the evolution. Of course we recover the typical breathing mode at $12.5 \mathrm{MeV}$ but also contributions around $17 \mathrm{MeV}$ and $26 \mathrm{MeV}$. The radial dependence of $\tilde{\rho}(r, \omega)$ for a given $\omega$ can be considered as the transition density of the corresponding mode. Therefore, the appearance of hole at the center of the nucleus seems to be connected with the surface waves. We have tested that removing the coulomb field does not modify the presented conclusions (as suggested in [23]). Therefore, the creation of a hollow 
structure is not a consequence of the coulomb interaction.

It should be noticed that starting from a value of $0.8, \rho_{2 f m} / \rho_{0}$ reaches much lower values during its evolution. Moreover, the minimum minimorum

(namely $\rho_{2 f m} / \rho_{0}=0.4$ ) is not reached during the first oscillation but at the third ones. The observed oscillation pattern of $\rho_{2 f m}$ is characteristic of the beating of several modes as clearly seen from the Fourier transform. It should be noticed that reducing the amplitude of the oscillations we have not observed a major modification of the Fourier spectrum. An example of such a case is shown in figure 3 (Bottom) for initial compression $\rho / \rho_{0}=1$. As far as the main peaks are concerned, the frequency structure of the Fourier transform does not change by more than a half $\mathrm{MeV}$ when the amplitude of the mode increased. However, looking in details we can see that the relative importance of the different modes is not the same. This is what we expect since the amplitude of each mode is directly related to the initialization procedure. This demonstrates that the coupling between modes and the anharmonicities are small, and that the main effect is the beatings of different modes. However, looking to the details of the radial structure of the Fourier transforms we can notice that the transition densities are slightly modified by the modification of the initial amplitude. This is clearly evidenced in figure 4 , where the transition densities $\tilde{\rho}(r, \omega)$ for the two main peaks $\omega=12 \mathrm{MeV}$ and $\omega=17 \mathrm{MeV}$ is displayed for the two initializations shown in figure 3 . In this figure we can see that the Giant monopole resonance around $12 \mathrm{MeV}$ is more robust than the smaller peak at $17 \mathrm{MeV}$ for which a contribution in the interior of the nucleus is seen at large amplitude. This volume contribution explains why a hollowed shape can appear as a function of time as the two modes are beating one again the other with different nodal structures.

\section{Conclusion}

In the presented simulation, the beatings of different monopole modes lead to very low densities in the interior of the nucleus and to the formation of a hollow structure after a long time (2 or 3 oscillation period). This tendency of excited nuclei to expand in hollow structures have already been observed in several dynamical calculations of the Vlasov type [22, 23] however the new feature shown by the presented quantum calculations is that this structure gets more pronounced after several collective oscillations. This analysis shows that this is due to the presence of several collective degrees of freedom with 
a transition density affecting the interior of the nucleus.

This could change the discussion about the possible fragmentation of nuclei and also about the involved time scales. Indeed, a possible interpretation of multifragmentation is that heavy excited system could reach dynamically a configuration in which the nucleus is unstable against a splitting in pieces. This might be in particular the case for low density regions or for hollow structures. In the framework of one collective variable picture, after preequilibrium, the excited nucleus reaches the lowest density region at the first turning-point of the collective expansion. This has given a widely admitted time-scale of this phenomenon[2] and is now often used to discuss break-up of a system 4 . If now we focus on $\rho_{2 \mathrm{fm}}$, we see that a nucleus could oscillate several times before reaching a configuration presenting density profiles with low density regions. If this configuration is unstable we see that the nucleus could wait a long time, of the order of $100 \mathrm{fm} / \mathrm{c}$ for the second turning-point or even $200 \mathrm{fm} / \mathrm{c}$ for the third, before initiating its fragmentation. It should be noticed that indications in direction of this scenario have been recently reported in hard photons study of Heavy-Ion collision 11.

Note also that we have observed the same behavior for different nuclei with higher temperatures. Moreover, we have applied the same analysis with a hard EOS (SIII force), and the same observations have been made. Therefore, the reported conclusion are rather generic. From the analysis we have related this behavior to the existence of a volume contribution with several nodes in the transition density. This, together with the Landau spreading, may occur as generic features and explains that the presented dynamics was found in many simulations.

In summary, we show that realistic TDHF calculations of expanding nuclei cannot be reduced to the simple large amplitude breathing mode picture. Generically, the observed dynamics presents the beating of several modes so that it may happen that low density regions can be reached after several global oscillations. This may have strong qualitative and quantitative consequences on the dynamical expansion of nuclei: in particular, time-scale could be strongly increased and exotic shapes can be reached.

\section{Acknowledgments}

We thank K. Bennaceur and G. Martinez for helpful discussions and M. Colonna for a careful reading of the manuscript. 


\section{References}

[1] G. Bertsch and P.J. Siemens, Phys. Lett. 126B (1983) 9.

[2] J. Cugnon, Phys. Lett. 135B (1986) 374.(and ref. therein.)

[3] W. A. Friedman, Phys. Rev. Lett. 60 (1988) 2125.

W. A. Friedman, Phys. Rev. C 42 (1990) 667.

[4] G. Papp and W. Nörenberg, International Workshop XXII on Gross Properties and Nuclear Excitation, Hirschegg, Austria, January, 17-22, 1994, p. 87. G. Papp and W. Nörenberg, Critical Phenomena and collective observables (CRIS'96), Acicastello, Italy, May 27-31, 1996, Ed. S. Costa et al, p. 377.

[5] For a review see J. Pochodzalla, Prog. Part. Nucl. Phys. 39 (1997) 443

[6] D.H.E. Gross, Rep. Prog. Phys. 53 (1990) 605.

J.P.Bondorf et al, Phys. Rep. 257 (1995) 133.

[7] M. Colonna and P. Chomaz, Phys. Rev. C49 (1994) 1908.

A. Guarnera, M. Colonna and P. Chomaz, Phys. Lett. B373 (1996) 267.

[8] S. Ayik and C. Gregoire, Phys. Lett. B 212, (1988) 269; Nucl. Phys. A513 (1990) 187.

[9] J. Randrup and B. Remaud, Nucl. Phys. A514 (1990) 339.

[10] G. Martinez et Al., Phys. Lett. B 349 (1995) 23

[11] For a review, see Y. Schutz et al, Preprint GANIL P 97 15, to be published in Nucl. Phys. A.

[12] N. Van Giai et al, Nucl. Phys.A482 (1988) 437c.

[13] P. Ring and P. Schuck, The Nuclear Many-Body Problem, Spring-Verlag, New-York (1980).

P. Chomaz and N. Frascaria, Phys. Rep. 252 (1995) 275.

[14] G.F. Bertsch and S. Das Gupta, Phys. Rep. 160 (1988) 189.

[15] D. Vautherin, J. Treiner and M. Veneroni, Phys. Lett. 191B (1987) 6. 
[16] D. Vautherin and D. M. Brink, Phys. Rev. C5 (1972) 626.

[17] S.E. Koonin and J.W. Negele, Phys. Rev. C15 (1977) 1359. K.T.R. Davies and S.E. Koonin, Phys. Rev. C23 (1981) 2042.

[18] P. Bonche, S. Koonin and J.W. Negele, Phys. Rec. C13 (1976) 1226.

[19] M. Brack, C. Guet and H. Håkansson, Phys. Rep. 123 (1985) 275.

[20] J.-J. Gaimard and K.-H. Schmidt, Nucl. Phys. A531 (1991) 709.

[21] J. Pochodzalla et al, Critical Phenomena and collective observables (CRIS'96), Acicastello, Italy, May 27-31, 1996, Ed. S. Costa et al, p. 1.

[22] W.Bauer et al, Phys. Rev. Lett. 69 (1992) 1988.

L.G.Moretto and G.J. Wozniak, Ann. Rev. Nucl. Part. Sc. 43 (1993) 379 .

[23] B. Borderie et al, Phys. Lett. B302 (1993) 15. 


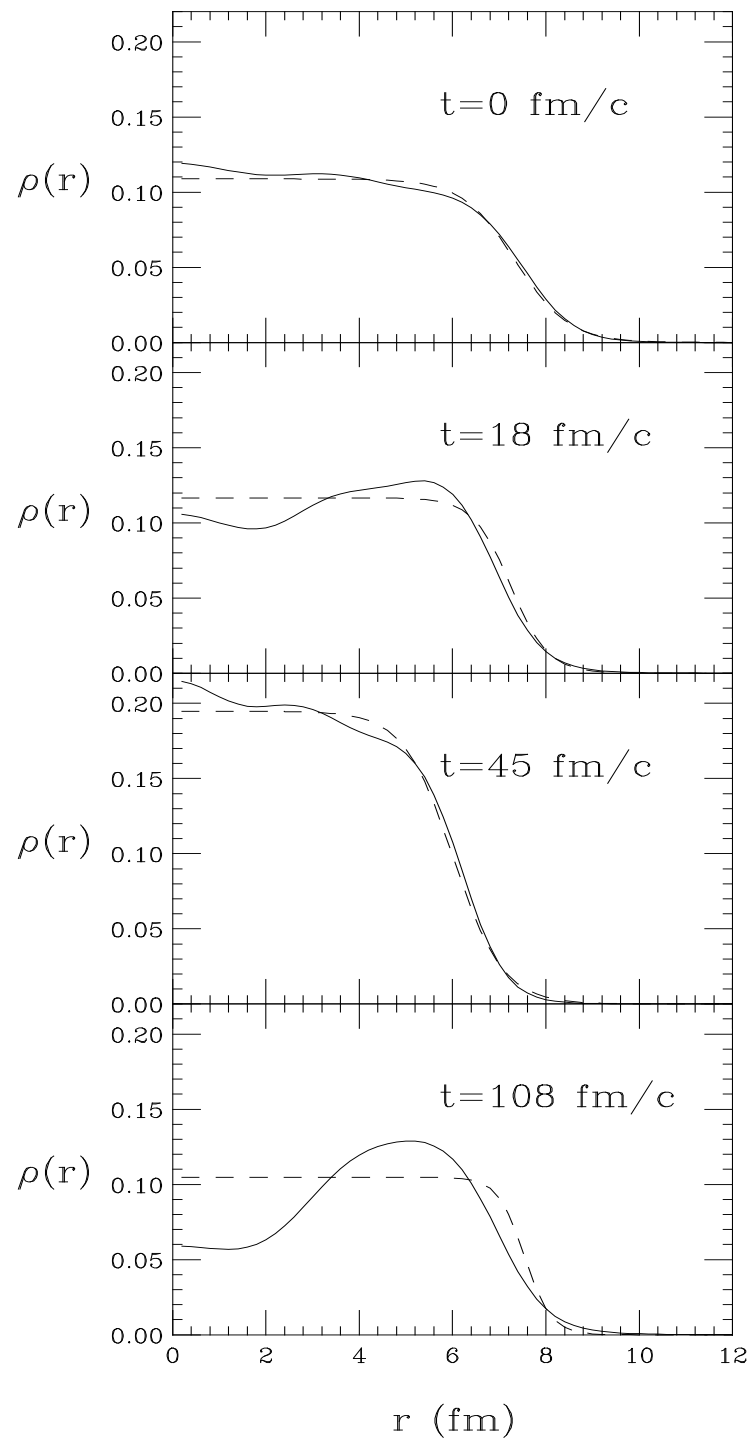

Figure 1: Density profile (solid curve) for different time. Dashed curve represent fits with a Fermi shape parametrisation of the density. This fit is not able to reproduce the complicated density profile (see $t=108 \mathrm{fm} / \mathrm{c}$ ) and demonstrate the difference between the TDHF evolution and the scaling picture. 


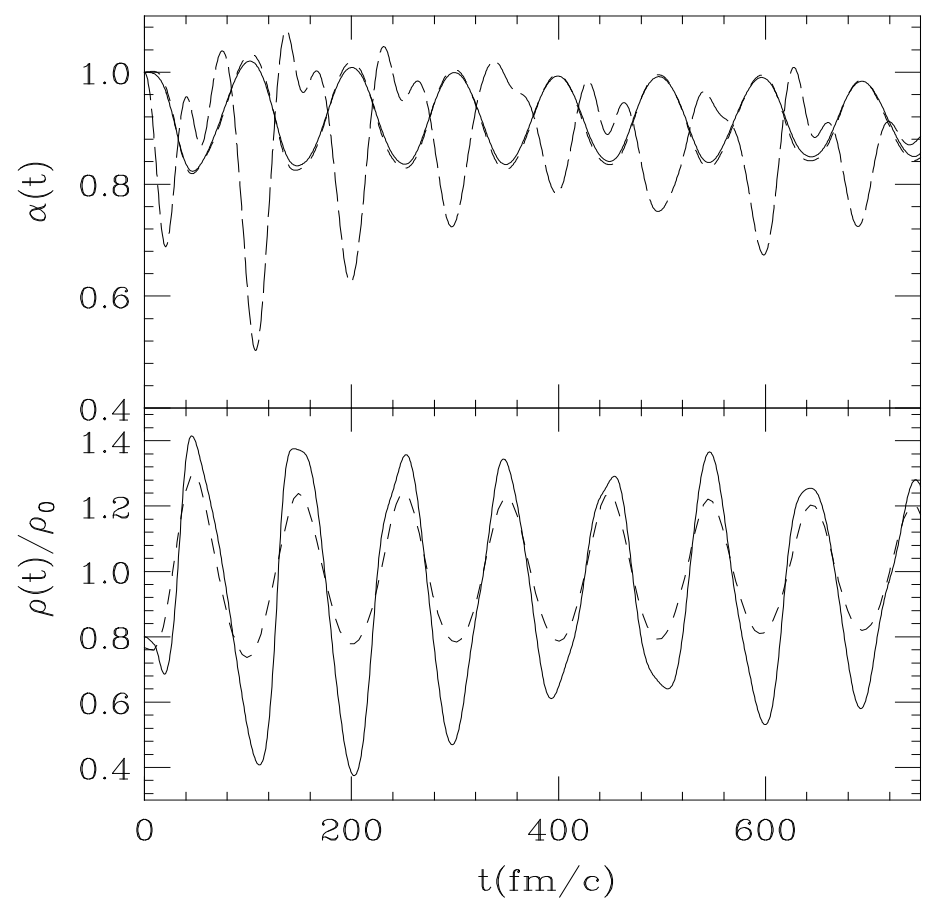

Figure 2: Top: Evolution of the three scaling factor calculated with the three parameters of the Fermi shape density. Dashed line: $\alpha_{a}$, long-dashed line: $\alpha_{R}$ and solid line: $\alpha_{\rho}$. Bot: Evolution of the central densitie $\rho_{2 f m}$ (solid curve) and $\rho_{5 \mathrm{fm}}$ (dashed curve) as a function of time. 


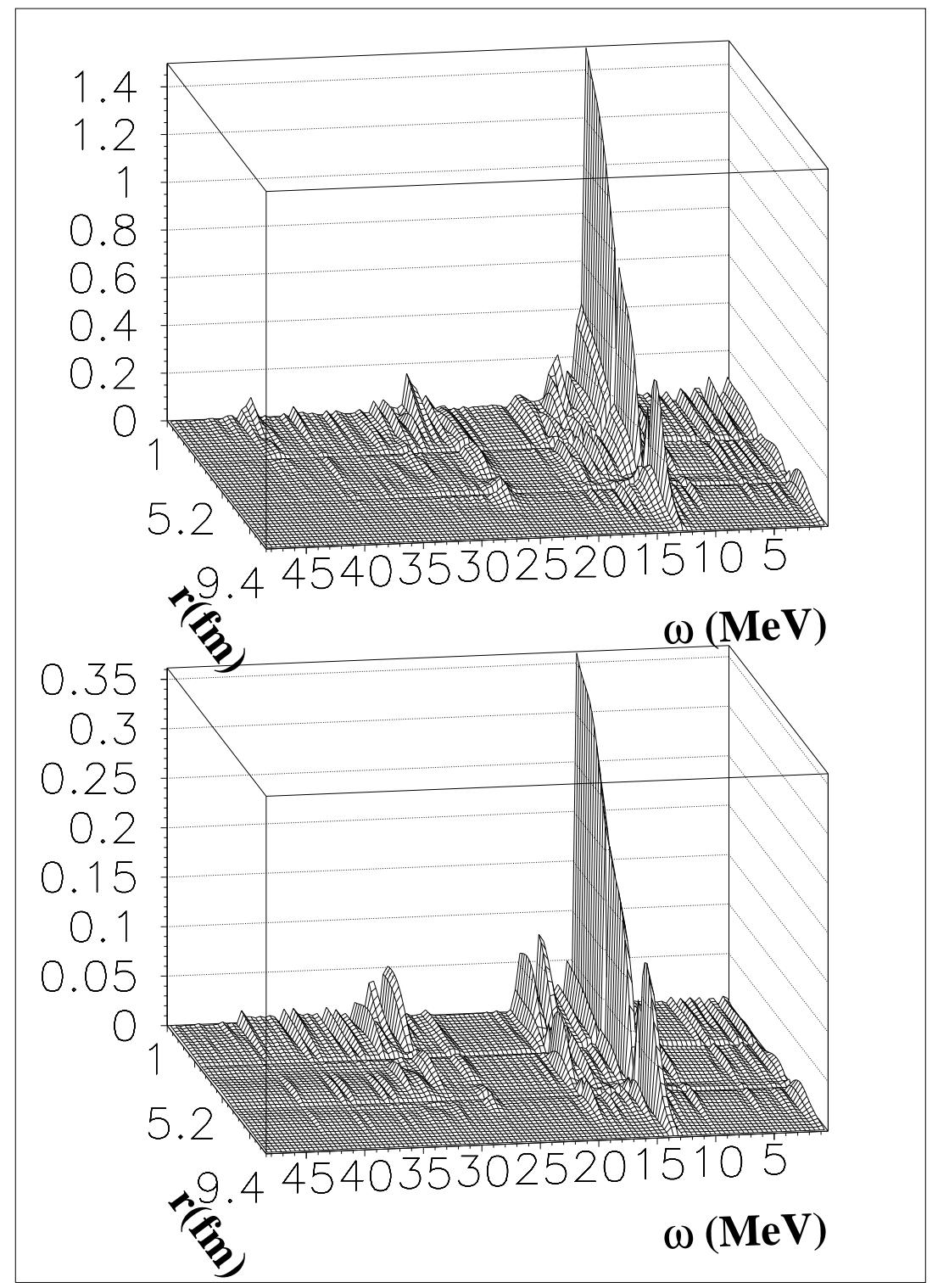

Figure 3: Fourier transform $|\tilde{\rho}(r, \omega)|$ (3D view). Two initial conditions are studied $\rho / \rho_{0}=0.8$ (top) and $\rho / \rho_{0}=1$ (bottom) are drawn. Fourier transforms are performed over $1500 \mathrm{fm} / \mathrm{c}$. 


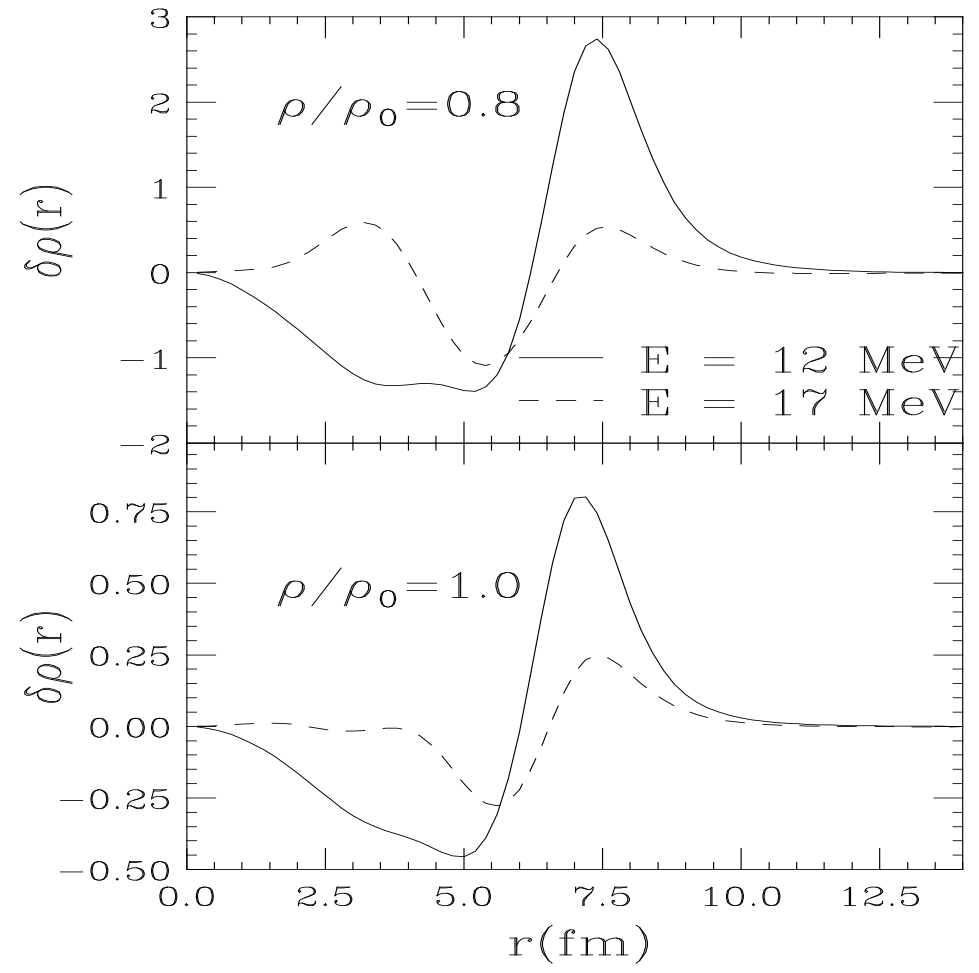

Figure 4: Transition densities $\tilde{\rho}(r, \omega)$ for $\omega=12 \mathrm{MeV}$ (solid line) and $\omega=17$ $\mathrm{MeV}$ (Dashed line) drawn for two values of the initial compression (see fig $3)$. 